\title{
EDITORIAL
}

\section{Igualdad de género y Derecho del trabajo y de la Seguridad Social: una integración inaplazable}

\author{
MARía Emilia CASAS BAAMONDE \\ Presidenta de la Asociación Española de Derecho \\ del Trabajo y de la Seguridad Social
}

doi: https://doi.org/10.20318/femeris.2019.4761

La decisión de la Asociación Española de Derecho del Trabajo y de la Seguridad Social de dotarse de una Comisión de Igualdad expresa su firme compromiso con la realización efectiva de la igualdad y la lucha contra las discriminaciones por cualquier causa "en el mundo de las relaciones laborales y de la Seguridad Social".

Conforme al artículo 2 de su Reglamento de funcionamiento, aprobado por la asamblea de la Asociación reunida el 31 de mayo de 2018 en la Universidad de Santiago de Compostela en el marco de su XVIII Congreso anual, son fines de la Comisión de Igualdad profundizar en la doctrina académica y en la renovación y divulgación del estudio y análisis sobre todas las formas de discriminación prohibidas en el ordenamiento interno, europeo e internacional, desempeñar una función divulgativa y formativa destinada a incrementar la conciencia social y el conocimiento acerca de las causas de discriminación prohibidas y los instrumentos jurídicos para combatirla, y proteger los derechos profesionales de las personas asociadas en materia de no discriminación. De acuerdo con el principio de transversalidad, ordena su Reglamento a la Comisión de Igualdad impulsar en las actividades de la Asociación la toma en consideración de la igualdad y de la no discriminación por causa del sexo y del género.

Se trata de promover la cultura de la igualdad con la convicción de que hoy no es posible abordar ningún proyecto, ni asociativo, ni investigador-docente, ni profesional de cualquier tipo, ni vital naturalmente, sin que en su realización se inserte el valor de la igualdad. Sin igualdad la Asociación no podría desempeñar sus cometidos de promover y difundir el estudio del Derecho del Trabajo y de la Seguridad Social en sus muy distintos ámbitos (académicos y profesionales; nacionales e internacionales), facilitar y promover la cooperación científica, así como colaborar en la defensa de los derechos fundamentales de las personas trabajadoras universalmente reconocidos; y no podría hacer suyas las necesidades de las personas a ella asociadas y de la sociedad. 
El trabajo de la Comisión Gestora de la Comisión de Igualdad a lo largo de este primer año de funcionamiento merece ser destacado y reconocido. La Junta Directiva de la Asociación desea hacerlo formalmente en estas páginas y expresar su agradecimiento a quienes la han integrado: Eva Blázquez Agudo, Fernando Lousada Arochena, Beatriz Quintanilla Navarro, Ricardo Esteban Legarreta, María Amparo Ballester Pastor, Antonio Álvarez del Cuvillo y Josefina Galindo Sánchez. En la página web de la Asociación pueden encontrar los lectores su carta de presentación.

La Comisión Gestora de la Comisión de Igualdad ha cumplido, entre otras, con su tarea de dotarse de instrumentos de divulgación de estudios y análisis en materia de igualdad. Promotora de una práctica de y en igualdad, sabe bien que esa práctica debe estar permanentemente orientada por una teoría avanzada animada en el análisis penetrante y crítico de la realidad y en la búsqueda de mecanismos adecuados y eficaces para corregir las desigualdades y lograr la equiparación sustancial de las personas y de los grupos en que se integran en sociedades diversas, y al tiempo cohesionadas, en que la igualdad no esté reñida con el respeto de las diferencias.

Gracias a la generosa iniciativa de la profesora Eva María Blázquez Agudo la Asociación ha celebrado un convenio de colaboración con la Universidad Carlos III de Madrid para publicar trabajos sobre el Derecho del Trabajo y de la Seguridad Social con perspectiva de género en un número extraordinario anual de la Revista FEMERIS, Revista Multidisciplinar de Estudios de Género que edita el Instituto Universitario de Estudios de Género de la citada Universidad. Lo ha expuesto perfectamente la profesora Blázquez, directora de la Revista y Vicedecana de Promoción, Orientación, Igualdad y Cooperación de su Facultad de Ciencias Sociales y Jurídicas, en su editorial "Sobre nuestra revista: un número extraordinario en materia de Derecho del Trabajo y de la Seguridad Social" de su volumen 4, no 1, de 2019. La Junta Directiva de la Asociación quiere agradecer a la profesora Blázquez su resuelta determinación en llevar a término esta iniciativa, que ofrece un valioso instrumento para la difusión de las investigaciones de género y del debate científico de nuestra disciplina, y que es hoy una feliz realidad.

La Revista FEMERIS tiene por objeto divulgar estudios doctrinales y rigurosos sobre género en el ámbito de todas las disciplinas jurídicas, sin perjuicio de ocuparse en general de la igualdad y la prohibición de discriminaciones. El Derecho del Trabajo y de la Seguridad Social, que tiene en su origen la desigualdad básica entre los trabajadores y los empresarios y en su finalidad su corrección, no puede concebirse en la actualidad de los Estados sociales y democráticos de Derecho sino como un proyecto global de construcción de la igualdad y, desde luego, de la género, sin la que no es posible el ejercicio de la libertad por las mujeres como personas y ciudadanas.

La división entre el trabajo remunerado y no remunerado por sexo conformó la disciplina que configura jurídicamente la realidad de aquel trabajo como un orden normativo autónomo, que en esa configuración respondió, como el ordenamiento jurídico en que se inserta, a relaciones sociales patriarcales y económicas basadas en el "hombre económico", provocando el "natural" aislamiento e invisibilidad de las mujeres en ambos tipos de trabajo, aunque relegadas al reproductivo y familiar no remunerado por disposición 
natural, en un mundo "naturalmente desigual". Si el "trabajador" estuvo ausente de las codificaciones civiles de la libertad e igualdad contractual, apenas aludido en los servicios que se pueden arrendar, las mujeres lo han estado de la construcción originaria de nuestra disciplina, dirigida a regular el intercambio patrimonial de trabajo por cuenta ajena y subordinado prestado por hombres y remunerado por hombres, titulares del poder económico y de los órganos de administración y decisión de las sociedades de capital. Las instituciones del Derecho colectivo se constituyeron sobre el mismo patrón antropológico masculino que ha dominado su funcionamiento durante demasiado tiempo.

La incorporación masiva de las mujeres al trabajo remunerado, hecho determinante e irreversible del pasado siglo, cambió decisivamente las cosas, exigiendo mecanismos de adecuación de las categorías jurídicas y de los dispositivos técnicos a la nueva realidad, que se han abierto paso con la lucha de las mujeres, graves dificultades conceptuales y resultados de discriminación e injusticia notorios. El cambio se convirtió en imparable cuando afectó a la percepción misma de la realidad sobre la que opera, demandado su reconstrucción a partir de la igualdad de género, referente objetivo de las transformaciones sociales. En su realización el Derecho en los distintos ámbitos en que se desenvuelve la actividad de las personas, y el Derecho del Trabajo y de la Seguridad Social en el suyo propio, se encuentran comprometidos, siendo la igualdad del género humano el fundamento mismo de su legitimación política democrática.

La ciencia del Derecho del Trabajo y de la Seguridad Social, y las políticas que han de plasmar sus soluciones al servicio del trabajo decente y del fortalecimiento de la protección social, han de partir de que la igualdad de género no puede ser una dimensión aislada, añadida desde fuera a su construcción nuclear y de sus instituciones propias, todavía sostenidas en la desigualdad de trato y de oportunidades y en la discriminación sistémica de las mujeres; al contrario, ha de ser un elemento definitorio desde dentro de esa construcción científica y política en un mundo global en el que han de ponerse a punto instrumentos normativos de igual alcance, hoy por hoy inexistentes, para el logro de la igualdad. En todo caso, han de darse por superados los tiempos del igualitarismo formal y de los valores abstractos, debiendo pasarse con decisión al logro de la igualdad real o sustancial de las personas, mujeres y hombres. En ello estamos, sin resignarnos a aceptar la predicción reciente del Fondo Monetario Internacional de que se precisará que pasen cien años para superar la brecha retributiva de género. Es lacerante el presagio de lentitud en la superación de una discriminación tan básica.

Este número de FEMERIS sirve a la elaboración del Derecho del Trabajo y de la Seguridad Social desde una teoría de la igualdad de género, que no puede perderse de vista salvo que la comprensión de la regulación de las relaciones laborales y de protección social se sitúe al margen de los procesos históricos de transformación, en la irrealidad más absoluta. Observación, razón crítica, sistematización, prospección y proposición van de la mano en los excelentes estudios que lo componen de la profesora María Amparo Ballester Pastor, del magistrado y profesor Fernando Lousada Arochena, y de las profesoras Carmen Sánchez Trigueros, Patricia Nieto Rojas, Concepción San Sáenz, Alejandra Selma Penalva, Gratiela-Florentina Moraru, y Loredana Ferluga. Siguen dos útiles crónicas de la 
jurisprudencia sobre igualdad, constitucional y europea, de los profesores Jean Jacqmain y Ron Latas, y a éstas provechosas reseñas de libros especializados de actualidad, en las que se comprueba la calidad y la pujanza de los profesores e investigadores que integran Sección Juvenil de la Asociación; en concreto, de Francisco Xabiere Gómez García, Macarena Ángel Quiroga y Francisco Miguel Ortiz González-Conde. Informa también FEMERIS de la segunda edición de la Jornada 8 Marzo de Igualdad de las Mujeres organizada por la Asociación el pasado 7 de marzo en colaboración con el Consejo Económico y Social y en su sede, en la que, junto con las contribuciones de las profesoras Sánchez Trigueros y Nieto Rojas, expuso su ponencia Esmeralda Sanz Berzal, sobre la promoción profesional de las mujeres en la negociación colectiva, como expresión del compromiso de los sindicatos y de las asociaciones empresariales con la igualdad de género y con la Asociación.

La lectura de este primer número extraordinario de FEMERIS hace evidente su ambición que augura el éxito de la iniciativa al servicio de la igualdad. Esto es lo que en verdad importa. 